International Mathematical Forum, Vol. 9, 2014, no. 36, 1779 - 1789

HIKARI Ltd, www.m-hikari.com

http://dx.doi.org/10.12988/imf.2014.411193

\title{
Iterative Algorithm for Common Minimum-Norm Fixed Point of a Finite Family of Asymptotically Nonextensive Nonself Mappings in Banach Spaces
}

\author{
Lijuan Zhang ${ }^{1}$ and Xian Wang
}

College of Mathematics and Information Science, Hebei University

Baoding, Hebei, 071002, P.R. China

Copyright (c) 2014 Lijuan Zhang and Xian Wang. This is an open access article distributed under the Creative Commons Attribution License, which permits unrestricted use, distribution, and reproduction in any medium, provided the original work is properly cited.

\begin{abstract}
We introduce an iterative algorithm for finding a common minimum norm fixed point of a finite family of asymptotically nonextensive nonself mappings. A strong convergence theorem of common element is established in a uniformly smooth and uniformly convex Banach space.
\end{abstract}

Mathematics Subject Classification: 47H09; 47H05

Keywords: Asymptotically nonextensive nonself mapping; Generalized projection operator; Minimum norm fixed point; Normalized duality mapping

\section{Introduction}

Let $H$ be a real Hilbert space, and $C$ be a nonempty closed convex subset of $H$. A mapping $S: C \rightarrow C$ be a nonexpansive mapping. $F(S)$ denotes the fixed point set of $S$. Iterative methods for finding fixed points of nonexpansive mappings are an important topic in the theory and have wide applications in a number of applied areas, such as, image reconstruction in computerized tomography, optics and neural networks, collective sensing etc. In general, the nonexpansive mapping may have more than one fixed point. Without loss of

\footnotetext{
${ }^{1}$ This work was supported by the Natural Science Foundation of Hebei Province (A2012201054) and the National Natural Science Foundation of China(11201110).
} 
generality, we may assume that $F(S) \neq \emptyset$. The minimum-norm fixed point of nonexpansive mappings is to find a fixed point of $S$ which satisfies:

$$
\bar{x} \in F(S):\|\bar{x}\|=\min \{\|x\|: x \in F(S)\} .
$$

That is, $\bar{x}$ is the minimum-norm fixed point of $S$. In other words, $\bar{x}$ is the metric projection of the origin into $F(S)$, i.e., $\bar{x}=P_{F(S)} 0$. It is an interesting thing to construct iterative sequence to find the minimum-norm fixed point of a nonexpansive mapping. Browder [1] introduced an implicit scheme as follows. Let $u \in C$ and $t \in(0,1), x_{t}$ be the unique fixed point in $C$ of the contraction $S_{t}$ from $C$ into $C$ :

$$
S_{t} x=t u+(1-t) S x, x \in C \text {. }
$$

Browder proved that the strong limit of $\left\{x_{t}\right\}$ as $t \rightarrow 0^{+}$is the fixed point of $S$ which is nearest from $F(S)$ to $u$. Halpern [2] introduced an explicit scheme. Let $u \in C$, define a sequence $\left\{x_{n}\right\}$ by the following:

$$
x_{n+1}=\alpha_{n} u+\left(1-\alpha_{n}\right) S x_{n}, n \geq 1 .
$$

Provided that $\left\{\alpha_{n}\right\} \subset(0,1)$ satisfies some conditions, Halpern proved that $\left\{x_{n}\right\}$ converges strongly to the fixed point of $S$ that is closest to $u$.

It is noticed that the methods studied above are used to approximate the minimum-norm fixed point of $S$ if $0 \in C$. In order to overcome the difficulties caused by possible exclusion of the origin from $C$. Yang et al. [3] introduced an explicit scheme given by

$$
x_{n+1}=\beta S x_{n}+(1-\beta) P_{C}\left[\left(1-\alpha_{n}\right) x_{n}\right], n \geq 1 .
$$

They proved that under appropriate conditions on $\left\{\alpha_{n}\right\}$ and $\beta$, the sequence $\left\{x_{n}\right\}$ converges strongly to minimum-norm fixed point of $S$. Yao and $\mathrm{Xu}$ [4] have shown that the explicit scheme

$$
x_{n+1}=P_{C}\left[\left(1-\alpha_{n}\right) S x_{n}\right], n \geq 1,
$$

the sequence $\left\{x_{n}\right\}$ converges strongly to minimum-norm fixed point of $S$ under appropriate conditions on $\left\{\alpha_{n}\right\}$.

Zegeye and Shahzad [5] introduced the iterative process which converges strongly to the common minimum-norm fixed point of a finite family of asymptotically nonexpansive mappings. They shows that the sequence $\left\{x_{n}\right\}$ defines by:

$$
\left\{\begin{array}{l}
x_{1} \in C \\
y_{n}=P_{C}\left[\left(1-\alpha_{n}\right) x_{n}\right] \\
x_{n+1}=\beta_{n, 0} x_{n}+\Sigma_{i=1}^{N} \beta_{n, i} S_{i}^{n} y_{n}, n \geq 1
\end{array}\right.
$$


Then $\left\{x_{n}\right\}$ converges strongly to the common minimum norm point. They extended Yang et al. [3] and Yao and $\mathrm{Xu}$ [4] from nonexpansive mappings to asymptotically nonexpansive mappings in Hilbert spaces.

Let $C$ be a nonempty subset of a real Banach space $E$. $S: C \rightarrow E$ is said to be asymptotically nonextensive nonself-mapping iff there exists a sequence $\left\{k_{n}\right\} \subset[1, \infty)$ with $\lim _{n \rightarrow \infty} k_{n}=1$, such that

$$
\phi\left(S\left(\Pi_{C} S\right)^{n-1} x, S\left(\Pi_{C} S\right)^{n-1} y\right) \leq k_{n} \phi(x, y), \quad \forall x, y \in C, \quad n \geq 1
$$

where $\Pi_{C}: E \rightarrow C$ is the generalized projection. Asymptotically nonextensive mappings coincide with asymptotically nonexpansive mappings in Hilbert spaces. For self-map in Hilbert spaces, the definition of asymptotically nonextensive nonself-mapping coincides with the definition of asymptotically nonexpansive mappings introduced by Goebel and Kirk [6]. In [7], Chidume, Khumalo and Zegeye first studied the fixed point problem of an asymptotically nonextensive nonself-mapping and obtained weak convergence theorem. Recently, Liu [8] introduced an iterative algorithm for a common fixed point problem of two asymptotically nonextensive nonself mappings.

A nature question arises whether we can extend the results of Zegeye and Shahzad [5] to a class of mappings more general than asymptotically nonexpansive mappings or not.

The purpose of this paper is to prove a strong convergence theorem for finding a common minimum-norm fixed point of a finite family of asymptotically nonextensive nonself mappings in a uniformly smooth and uniformly convex Banach space.

\section{Preliminaries}

Let $E$ be a real Banach space with norm $\|$.$\| , let E^{*}$ denote the dual of $E$ and let $\langle x, f\rangle$ denote the value of $f \in E^{*}$ at $x \in E$. When $\left\{x_{n}\right\}$ is a sequence in $E$, we denote strong convergence of $\left\{x_{n}\right\}$ to $x \in E$ by $x_{n} \rightarrow x$ and weak convergence by $x_{n} \rightarrow x$. Suppose that $C$ is a nonempty, closed convex subset of $E$. Let $J$ be the normalized duality mapping from $E$ into $2^{E^{*}}$ given by

$$
J x=\left\{f \in E^{*}:\langle x, f\rangle=\|x\|\|f\|,\|x\|=\|f\|\right\}
$$

for all $x \in E$. It is well known that if $E$ is uniformly smooth, then $J$ is uniformly norm-to-norm continuous on each bounded subset of $E$. It is also well known that $E$ is uniformly smooth if and only if $E^{*}$ is uniformly convex. $E$ is said to have the Kadec-Klee property iff for a sequence $\left\{x_{n}\right\}$ of $E$ satisfying that $x_{n} \rightarrow x \in E$ and $\left\|x_{n}\right\| \rightarrow\|x\|$, then $x_{n} \rightarrow x$. It is known that if $E$ is uniformly convex, then $E$ has the Kadec-Klee property. 
Let $C$ is a nonempty closed convex subset of a Hilbert space $H$ and $P_{C}$ : $H \rightarrow C$ is the metric projection of $H$ onto $C$, then $P_{C}$ is nonexpansive. This fact actually characterizes Hilbert spaces and consequently, it is not available in more general Banach spaces. In this connection, Alber [9] recently introduced a generalized projection operator $\Pi_{C}$ in a smooth, strictly, reflexive Banach space $E$ which is an analogue of the metric projection in Hilbert spaces. Consider the functional defined by

$$
\phi(x, y)=\|x\|^{2}-2\langle x, J y\rangle+\|y\|^{2}, \forall x, y \in E .
$$

Observe that, in a Hilbert space $H,(2.2)$ reduces to $\phi(x, y)=\|x-y\|^{2}, x, y \in$ $H$. The generalized projection $\Pi_{C}: E \rightarrow C$ is a map that assigns to an arbitrary point $x \in E$ the minimum point of the functional $\phi(x, y)$, that is, $\Pi_{C} x=\bar{x}$, where $\bar{x}$ is the solution to the minimization problem

$$
\phi(\bar{x}, x)=\min _{y \in C} \phi(y, x),
$$

existence and uniqueness of the operator $\Pi_{C}$ follows from the properties of the functional $\phi(x, y)$ and strict monotonicity of the mapping $J$. In Hilbert spaces, $\Pi_{C}=P_{C}$. It is obvious from the definition of function $\phi$ that

$$
\begin{gathered}
(\|x\|-\|y\|)^{2} \leq \phi(y, x) \leq(\|x\|+\|y\|)^{2}, \forall x, y \in E . \\
\phi(x, y)=\phi(x, z)+\phi(z, y)+2\langle x-z, J z-J y\rangle, \forall x, y, z \in E . \\
\phi(x, y)=\langle x, J x-J y\rangle+\langle y-x, J y\rangle \leq\|x\|\|J x-J y\|+\|y-x\|\|y\|, \forall x, y \in E . \\
\phi\left(x, J^{-1}(\lambda J y+(1-\lambda) J z)\right) \leq \lambda \phi(x, y)+(1-\lambda) \phi(x, z), \forall x, y, z \in E, \forall \lambda \in(0,1) .
\end{gathered}
$$

In order to prove our main result, we need the following Lemma.

Lemma 2.1 [8] Let $E$ be a uniformly smooth and strictly convex Banach space which satisfies the Kadec-Klee property. Let $C$ be a nonempty, closed and convex subset of $E$. If $S: C \rightarrow E$ be an asymptotically nonextensive nonself-mapping with a sequence $\left\{k_{n}\right\} \subset[1, \infty)$ such that $I-S$ is demi-closed at zero, then $F(S)$ is closed and convex.

Lemma 2.2 [9] Let $E$ be a reflexive, strictly convex and smooth Banach space, let $C$ be a nonempty, closed and convex subset of $E$. Then the following conclusions hold:

(1) $\phi\left(y, \Pi_{C} x\right)+\phi\left(\Pi_{C} x, x\right) \leq \phi(y, x), \forall y \in C$, and $x \in E$;

(2) If $x \in E$ and $z \in C$, Then $z=\Pi_{C} x$ if and only if $\langle z-y, J x-J z\rangle \geq$ $0, \forall y \in C$;

(3) For $x, y \in E, \phi(y, x)=0$ if and only if $x=y$.

Lemma $2.3[10]$ Let $E$ be a uniformly convex and smooth Banach space and let $\left\{y_{n}\right\},\left\{z_{n}\right\}$ be two sequences of $E$. If $\phi\left(y_{n}, z_{n}\right) \rightarrow 0$, and either $\left\{y_{n}\right\}$, or $\left\{z_{n}\right\}$ is bounded, then $y_{n}-z_{n} \rightarrow 0$. 
Lemma 2.4 [11] Let $E$ be a uniformly convex and smooth Banach space and let $r>0$. Then there exists a continuous, strictly increasing and convex function $g:[0,2 r] \rightarrow R$ such that $g(0)=0$ and

$$
\|t x+(1-t) y\|^{2} \leq t\|x\|^{2}+(1-t)\|y\|^{2}-t(1-t) g(\|x-y\|),
$$

for all $x, y \in B_{r}$ and $t \in[0,1]$, where $B_{r}:=\{z \in E:\|z\| \leq r\}$.

We make use of the function $V: E \times E^{*} \rightarrow R$ defined by

$$
V\left(x, x^{*}\right)=\|x\|^{2}-2\left\langle x, x^{*}\right\rangle+\left\|x^{*}\right\|^{2}, x \in E, x^{*} \in E^{*}
$$

which was studied by Alber [9]. That is, $V\left(x, x^{*}\right)=\phi\left(x, J^{-1} x^{*}\right)$ for all $x \in E$ and $x^{*} \in E^{*}$. We know the following Lemma.

Lemma 2.5 [9] Let $E$ be a reflexive, strictly convex and smooth Banach space with $E^{*}$ as its dual. Then

$$
V\left(x, x^{*}\right)+2\left\langle J^{-1} x^{*}-x, y^{*}\right\rangle \leq V\left(x, x^{*}+y^{*}\right)
$$

for all $x \in E$ and $x^{*}, y^{*} \in E^{*}$.

Lemma 2.6 [12] Let $\left\{s_{n}\right\}$ be a sequence of nonnegative real numbers such that:

$$
s_{n+1} \leq\left(1-\lambda_{n}\right) s_{n}+\beta_{n}, n \geq 0,
$$

where $\left\{\lambda_{n}\right\},\left\{\beta_{n}\right\}$ satisfy the conditions:

(i) $\left\{\lambda_{n}\right\} \subset(0,1)$ and $\sum_{n=1}^{\infty} \lambda_{n}=\infty$,

(ii) $\lim \sup _{n \rightarrow \infty} \frac{\beta_{n}}{\lambda_{n}} \leq 1$. Then $\lim _{n \rightarrow \infty} s_{n}=0$.

Lemma 2.7 [13] Let $\left\{a_{n}\right\}$ be a sequence of real numbers such that there exists a subsequence $\left\{n_{i}\right\}$ of $\{n\}$ such that $a_{n_{i}} \leq a_{n_{i}+1}$ for all $i \in \mathbf{N}$. Then there exists a nondecreasing sequence $\left\{m_{k}\right\} \subset \mathbf{N}$ such that $m_{k} \rightarrow \infty$ and the following properties are satisfied by all numbers for all $k \in \mathbf{N}$ :

$$
a_{m_{k}} \leq a_{m_{k}+1} \text { and } a_{k} \leq a_{m_{k}+1} .
$$

In fact, $\left\{m_{k}\right\}=\max \left\{j \leq k: a_{j}<a_{j+1}\right\}$.

\section{Main Results}

Theorem 3.1 let $C$ be a nonempty closed convex subset of a uniformly smooth and uniformly convex Banach space $E$. Let $S_{i}: C \rightarrow E$ be asymptotically nonextensive nonself mapping with sequence $\left\{k_{n, i}\right\}$ for each $1 \leq i \leq N$. Suppose that $I-S_{i}$ is demi-closed and $F=\bigcap_{i=1}^{N} F\left(S_{i}\right)$ is nonempty. Let $\left\{\alpha_{n}\right\}$, $\left\{\beta_{n, i}\right\}$ be the sequences in $(0,1)$ satisfying the following conditions:

$$
\lim _{n \rightarrow \infty} \alpha_{n}=0, \sum_{n=1}^{\infty} \alpha_{n}=\infty ; \lim _{n \rightarrow \infty} \frac{k_{n, i}-1}{\alpha_{n}}=0, \forall 1 \leq i \leq N ;
$$


$\liminf _{n \rightarrow \infty} \beta_{n, 0} \beta_{n, i}>0, \forall 1 \leq i \leq N ; \sum_{i=0}^{N} \beta_{n, i}=1, \forall n \geq 1$.

Let $\left\{x_{n}\right\}$ be a sequence generated by the following manner:

$$
\left\{\begin{array}{c}
x_{1} \in C \\
y_{n}=\Pi_{C}\left[\left(1-\alpha_{n}\right) x_{n}\right] \\
x_{n+1}=J^{-1}\left(\beta_{n, 0} J x_{n}+\sum_{i=1}^{N} \beta_{n, i} J S_{i}\left(\Pi_{C} S_{i}\right)^{n-1} y_{n}\right)
\end{array}\right.
$$

Then $\left\{x_{n}\right\}$ converges strongly to the common minimum norm point of $F$.

Proof Let $\bar{x}=\Pi_{F}(0) \in F$. That is $\|\bar{x}\|^{2}=\phi(\bar{x}, 0)=\min _{y \in F} \phi(y, 0)=$ $\min _{y \in F}\|y\|^{2}$.

Let $k_{n}=\max _{1 \leq i \leq N}\left\{k_{n, i}\right\}$. Then from (3.1) and Lemma 2.2, we have that

$$
\begin{aligned}
\phi\left(\bar{x}, y_{n}\right) & =\phi\left(\bar{x}, \Pi_{C}\left(\left(1-\alpha_{n}\right) x_{n}\right)\right) \\
& \leq \phi\left(\bar{x},\left(1-\alpha_{n}\right) x_{n}\right) \\
& =\|\bar{x}\|^{2}-2\left\langle\bar{x}, J\left(1-\alpha_{n}\right) x_{n}\right\rangle+\left\|\left(1-\alpha_{n}\right) x_{n}\right\|^{2} \\
& \leq\|\bar{x}\|^{2}-2\left\langle\bar{x},\left(1-\alpha_{n}\right) J x_{n}\right\rangle+\left(1-\alpha_{n}\right)\left\|x_{n}\right\|^{2} \\
& \leq \alpha_{n}\|\bar{x}\|^{2}+\left(1-\alpha_{n}\right) \phi\left(\bar{x}, x_{n}\right) .
\end{aligned}
$$

From (2.6) and (3.2), we have that

$$
\begin{aligned}
\phi\left(\bar{x}, x_{n+1}\right) & =\phi\left(\bar{x}, J^{-1}\left(\beta_{n, 0} J x_{n}+\sum_{i=1}^{N} \beta_{n, i} J S_{i}\left(\Pi_{C} S_{i}\right)^{n-1} y_{n}\right)\right. \\
& \leq \beta_{n, 0} \phi\left(\bar{x}, x_{n}\right)+\sum_{i=1}^{N} \beta_{n, i} \phi\left(\bar{x}, S_{i}\left(\prod_{C} S_{i}\right)^{n-1} y_{n}\right) \\
& \leq \beta_{n, 0} \phi\left(\bar{x}, x_{n}\right)+\sum_{i=1}^{N} \beta_{n, i} k_{n, i} \phi\left(\bar{x}, y_{n}\right) \\
& \leq \beta_{n, 0} \phi\left(\bar{x}, x_{n}\right)+\sum_{i=1}^{N} \beta_{n, i} k_{n} \phi\left(\bar{x}, y_{n}\right) \\
& \leq \beta_{n, 0} \phi\left(\bar{x}, x_{n}\right)+\left(1-\beta_{n, 0}\right) k_{n}\left[\alpha_{n}\|\bar{x}\|^{2}+\left(1-\alpha_{n}\right) \phi\left(\bar{x}, x_{n}\right)\right] \\
& \leq\left[\beta_{n, 0}+\left(1-\beta_{n, 0}\right) k_{n}\left(1-\alpha_{n}\right)\right] \phi\left(\bar{x}, x_{n}\right)+\left(1-\beta_{n, 0}\right) k_{n} \alpha_{n}\|\bar{x}\|^{2} \\
& \leq \delta_{n}\|\bar{x}\|^{2}+\left[1-(1-\varepsilon) \delta_{n}\right] \phi\left(\bar{x}, x_{n}\right),
\end{aligned}
$$

where $\delta_{n}=\left(1-\beta_{n, 0}\right) k_{n} \alpha_{n}$. Since there exists $N_{0}>0$ such that $\frac{k_{n}-1}{\alpha_{n}}<\varepsilon k_{n}$ for all $n \geq N_{0}$ and for some $1>\varepsilon>0$ satisfying $(1-\varepsilon) \delta_{n}<1$. $\stackrel{\alpha_{n}}{\text { Then, by }}$ introduction

$$
\phi\left(\bar{x}, x_{n+1}\right) \leq \max \left\{(1-\varepsilon)^{-1}\|\bar{x}\|^{2}, \phi\left(\bar{x}, x_{0}\right)\right\}
$$

which implies that $\left\{x_{n}\right\}$ and $\left\{y_{n}\right\}$ are bounded. Moreover from (2.6) and Lemma 2.5, we obtain that

$$
\begin{aligned}
\phi\left(\bar{x}, y_{n}\right) & \leq \phi\left(\bar{x},\left(1-\alpha_{n}\right) x_{n}\right) \\
& =V\left(\bar{x}, J\left(1-\alpha_{n}\right) x_{n}\right) \\
& \leq V\left(\bar{x}, J\left(1-\alpha_{n}\right) x_{n}+\alpha_{n} J \bar{x}\right)-2\left\langle J^{-1} J\left(1-\alpha_{n}\right) x_{n}-\bar{x}, \alpha_{n} J \bar{x}\right\rangle \\
& =\phi\left(\bar{x}, J^{-1}\left(J\left(1-\alpha_{n}\right) x_{n}+\alpha_{n} J \bar{x}\right)\right)-2\left\langle\left(1-\alpha_{n}\right) x_{n}-\bar{x}, \alpha_{n} J \bar{x}\right\rangle \\
& \leq\left(1-\alpha_{n}\right) \phi\left(\bar{x}, x_{n}\right)+\alpha_{n} \phi(\bar{x}, \bar{x})-2 \alpha_{n}\left\langle\left(1-\alpha_{n}\right) x_{n}-\bar{x}, J \bar{x}\right\rangle \\
& =\left(1-\alpha_{n}\right) \phi\left(\bar{x}, x_{n}\right)-2 \alpha_{n}\left\langle\left(1-\alpha_{n}\right) x_{n}-\bar{x}, J \bar{x}\right\rangle .
\end{aligned}
$$


Since $E$ is a uniformly smooth Banach space, we know that $E^{*}$ is a uniformly convex Banach space. From Lemma 2.4, we find that

$$
\begin{aligned}
& \phi\left(\bar{x}, x_{n+1}\right) \\
& =\|\bar{x}\|^{2}-2\left\langle\bar{x}, \beta_{n, 0} J x_{n}+\sum_{i=1}^{N} \beta_{n, i} J S_{i}\left(\Pi_{C} S_{i}\right)^{n-1} y_{n}\right\rangle+\left\|\beta_{n, 0} J x_{n}+\sum_{i=1}^{N} \beta_{n, i} J S_{i}\left(\Pi_{C} S_{i}\right)^{n-1} y_{n}\right\|^{2} \\
& \leq\|\bar{x}\|^{2}-2 \beta_{n, 0}\left\langle\bar{x}, J x_{n}\right\rangle-2 \sum_{i=1}^{N} \beta_{n, i}\left\langle\bar{x}, J S_{i}\left(\Pi_{C} S_{i}\right)^{n-1} y_{n}\right\rangle \\
& +\beta_{n, 0}\left\|x_{n}\right\|^{2}+\sum_{i=1}^{N} \beta_{n, i}\left\|S_{i}\left(\Pi_{C} S_{i}\right)^{n-1} y_{n}\right\|^{2}-\sum_{i=1}^{N} \beta_{n, 0} \beta_{n, i} g\left(\left\|J x_{n}-J S_{i}\left(\Pi_{C} S_{i}\right)^{n-1} y_{n}\right\|\right) \\
& =\beta_{n, 0} \phi\left(\bar{x}, x_{n}\right)+\sum_{i=1}^{N} \beta_{n, i} \phi\left(\bar{x}, S_{i}\left(\Pi_{C} S_{i}\right)^{n-1} y_{n}\right)-\sum_{i=1}^{N} \beta_{n, 0} \beta_{n, i} g\left(\left\|J x_{n}-J S_{i}\left(\Pi_{C} S_{i}\right)^{n-1} y_{n}\right\|\right) \\
& \leq \beta_{n, 0} \phi\left(\bar{x}, x_{n}\right)+\sum_{i=1}^{N} \beta_{n, i} k_{n, i} \phi\left(\bar{x}, y_{n}\right)-\sum_{i=1}^{N} \beta_{n, 0} \beta_{n, i} g\left(\left\|J x_{n}-J S_{i}\left(\Pi_{C} S_{i}\right)^{n-1} y_{n}\right\|\right) \\
& \leq \beta_{n, 0} \phi\left(\bar{x}, x_{n}\right)+\sum_{i=1}^{N} \beta_{n, i} k_{n} \phi\left(\bar{x}, y_{n}\right)-\sum_{i=1}^{N} \beta_{n, 0} \beta_{n, i} g\left(\left\|J x_{n}-J S_{i}\left(\Pi_{C} S_{i}\right)^{n-1} y_{n}\right\|\right) .
\end{aligned}
$$

Using (3.4), we have that

$$
\begin{aligned}
& \phi\left(\bar{x}, x_{n+1}\right) \\
& \leq \beta_{n, 0} \phi\left(\bar{x}, x_{n}\right)+\left(1-\beta_{n, 0}\right) k_{n} \phi\left(\bar{x}, y_{n}\right)-\Sigma_{i=1}^{N} \beta_{n, 0} \beta_{n, i} g\left(\left\|J x_{n}-J S_{i}\left(\Pi_{C} S_{i}\right)^{n-1} y_{n}\right\|\right) \\
& \leq \beta_{n, 0} \phi\left(\bar{x}, x_{n}\right)+\left(1-\beta_{n, 0}\right)\left[\left(1-\alpha_{n}\right) \phi\left(\bar{x}, x_{n}\right)-2 \alpha_{n}\left\langle\left(1-\alpha_{n}\right) x_{n}-\bar{x}, J \bar{x}\right\rangle\right] \\
& -\sum_{i=1}^{N} \beta_{n, 0} \beta_{n, i} g\left(\left\|J x_{n}-J S_{i}\left(\Pi_{C} S_{i}\right)^{n-1} y_{n}\right\|\right)+\left(1-\beta_{n, 0}\right)\left(k_{n}-1\right) \phi\left(\bar{x}, y_{n}\right) \\
& \leq\left(1-\theta_{n}\right) \phi\left(\bar{x}, x_{n}\right)-2 \theta_{n}\left\langle\left(1-\alpha_{n}\right) x_{n}-\bar{x}, J \bar{x}\right\rangle \\
& -\sum_{i=1}^{N} \beta_{n, 0} \beta_{n, i} g\left(\left\|J x_{n}-J S_{i}\left(\Pi_{C} S_{i}\right)^{n-1} y_{n}\right\|\right)+\left(k_{n}-1\right) M \\
& \leq\left(1-\theta_{n}\right) \phi\left(\bar{x}, x_{n}\right)-2 \theta_{n}\left\langle\left(1-\alpha_{n}\right) x_{n}-\bar{x}, J \bar{x}\right\rangle+\left(k_{n}-1\right) M .
\end{aligned}
$$

for some $M>0$, where $\theta_{n}=\alpha_{n}\left(1-\beta_{n, 0}\right)$ for all $n \geq 1$. Note that $\lim _{n \rightarrow \infty} \theta_{n}=$ $0, \sum_{n=1}^{\infty} \theta_{n}=\infty$.

Now, the rest of proof is divided into two parts.

Case 1. Suppose that there exist $n_{0} \in \mathbf{N}$ such that $\left\{\phi\left(\bar{x}, x_{n}\right)\right\}$ is nonincreasing for all $n \geq n_{0}$. In this situation, $\left\{\phi\left(\bar{x}, x_{n}\right)\right\}$ is convergent. Then from (3.5), we have that

$$
\sum_{i=1}^{N} \beta_{n, 0} \beta_{n, i} g\left(\left\|J x_{n}-J S_{i}\left(\Pi_{C} S_{i}\right)^{n-1} y_{n}\right\|\right) \rightarrow 0, n \rightarrow \infty .
$$

From $\liminf _{n \rightarrow \infty} \beta_{n, 0} \beta_{n, i}>0$, we have that

$$
g\left(\left\|J x_{n}-J S_{i}\left(\Pi_{C} S_{i}\right)^{n-1} y_{n}\right\|\right) \rightarrow 0, n \rightarrow \infty .
$$

Therefore, from the property of $g$, we have that

$$
\lim _{n \rightarrow \infty}\left\|J x_{n}-J S_{i}\left(\Pi_{C} S_{i}\right)^{n-1} y_{n}\right\|=0, \forall 1 \leq i \leq N .
$$

Since $J^{-1}$ is uniformly norm-to-norm continuous on each bounded set, we have

$$
\lim _{n \rightarrow \infty}\left\|x_{n}-S_{i}\left(\Pi_{C} S_{i}\right)^{n-1} y_{n}\right\|=0 .
$$

Using (2.5), (3.6) and (3.7), we get that

$$
\lim _{n \rightarrow \infty} \phi\left(x_{n}, S_{i}\left(\Pi_{C} S_{i}\right)^{n-1} y_{n}\right)=0 .
$$


Moreover, from (3.8)

$$
\begin{aligned}
\phi\left(x_{n}, x_{n+1}\right) & =\phi\left(\bar{x}, J^{-1}\left[\beta_{n, 0} J x_{n}+\sum_{i=1}^{N} \beta_{n, i} J S_{i}\left(\Pi_{C} S_{i}\right)^{n-1} y_{n}\right)\right. \\
& \leq \beta_{n, 0} \phi\left(x_{n}, x_{n}\right)+\sum_{i=1}^{N} \beta_{n, i} \phi\left(\bar{x}, S_{i}\left(\Pi_{C} S_{i}\right)^{n-1} y_{n}\right) \\
& \leq \Sigma_{i=1}^{N} \beta_{n, i} \phi\left(\bar{x}, S_{i}\left(\Pi_{C} S_{i}\right)^{n-1} y_{n}\right) \rightarrow 0, n \rightarrow \infty
\end{aligned}
$$

From $\lim _{n \rightarrow \infty} \alpha_{n}=0$, we obtain that

$$
\begin{aligned}
\phi\left(x_{n}, y_{n}\right) & =\phi\left(x_{n}, \Pi_{C}\left(\left(1-\alpha_{n}\right) x_{n}\right)\right) \\
& \leq \phi\left(x_{n},\left(1-\alpha_{n}\right) x_{n}\right) \\
& \leq\left\|x_{n}\right\|^{2}-2\left\langle x_{n}, J\left(1-\alpha_{n}\right) x_{n}\right\rangle+\left\|\left(1-\alpha_{n}\right) x_{n}\right\|^{2} \\
& \leq\left\|x_{n}\right\|^{2}-2\left\langle x_{n},\left(1-\alpha_{n}\right) J x_{n}\right\rangle+\left(1-\alpha_{n}\right)\left\|x_{n}\right\|^{2} \\
& \leq \alpha_{n}\left\|x_{n}\right\|^{2} \rightarrow 0, n \rightarrow \infty .
\end{aligned}
$$

By Lemma 2.3 and (3.8-3.10), we have that

$$
\lim _{n \rightarrow \infty} x_{n}-S_{i}\left(\Pi_{C} S_{i}\right)^{n-1} y_{n}=0, \lim _{n \rightarrow \infty} x_{n}-y_{n}=0, \lim _{n \rightarrow \infty} x_{n}-x_{n+1}=0 .
$$

Furthermore $J$ is uniformly norm-to-norm continuous on each bounded set,

$$
\begin{gathered}
\lim _{n \rightarrow \infty} y_{n}-S_{i}\left(\Pi_{C} S_{i}\right)^{n-1} y_{n}=0, \lim _{n \rightarrow \infty} J y_{n}-J S_{i}\left(\Pi_{C} S_{i}\right)^{n-1} y_{n}=0, \\
\lim _{n \rightarrow \infty} y_{n}-y_{n+1}=0, \lim _{n \rightarrow \infty} J y_{n}-J y_{n+1}=0 .
\end{gathered}
$$

From (2.5),

$$
\lim _{n \rightarrow \infty} \phi\left(y_{n}, y_{n+1}\right)=0, \lim _{n \rightarrow \infty} \phi\left(y_{n}, S_{i}\left(\Pi_{C} S_{i}\right)^{n-1} y_{n}\right)=0, \forall 1 \leq i \leq N
$$

By (2.4), we obtain

$$
\begin{aligned}
& \phi\left(y_{n}, S_{1} y_{n}\right)=\phi\left(y_{n}, y_{n+1}\right)+\phi\left(y_{n+1}, S_{1} y_{n}\right)+2\left\langle y_{n}-y_{n+1}, J y_{n+1}-J S_{1} y_{n}\right\rangle \\
& =\phi\left(y_{n}, y_{n+1}\right)+\phi\left(y_{n+1}, S_{1}\left(\Pi_{C} S_{1}\right)^{n} y_{n+1}\right)+\phi\left(S_{1}\left(\Pi_{C} S_{1}\right)^{n} y_{n+1}, S_{1}\left(\Pi_{C} S_{1}\right)^{n} y_{n}\right) \\
& +2\left\langle S_{1}\left(\Pi_{C} S_{1}\right)^{n} y_{n+1}-S_{1}\left(\Pi_{C} S_{1}\right)^{n} y_{n}, J S_{1}\left(\Pi_{C} S_{1}\right)^{n} y_{n}-J S_{1} y_{n}\right\rangle \\
& +2\left\langle y_{n+1}-S_{1}\left(\Pi_{C} S_{1}\right)^{n} y_{n+1}, J S_{1}\left(\Pi_{C} S_{1}\right)^{n} y_{n+1}-J S_{1} y_{n}\right\rangle \\
& +2\left\langle y_{n}-y_{n+1}, J y_{n+1}-J S_{1} y_{n}\right\rangle+\phi\left(S_{1}\left(\Pi_{C} S_{1}\right)^{n} y_{n}, S_{1} y_{n}\right) .
\end{aligned}
$$

Since $\phi\left(y_{n},\left(\Pi_{C} S_{1}\right)^{n} y_{n}\right) \leq \phi\left(y_{n}, S_{1}\left(\Pi_{C} S_{1}\right)^{n-1} y_{n}\right)$, from (3.12), we have

$\lim _{n \rightarrow \infty} \phi\left(y_{n},\left(\Pi_{C} S_{1}\right)^{n} y_{n}\right)=0$. By Lemma 2.3, $\lim _{n \rightarrow \infty}\left\|y_{n}-\left(\Pi_{C} S_{1}\right)^{n} y_{n}\right\|=0$. Furthermore, we have $\lim _{n \rightarrow \infty} \phi\left(\left(\Pi_{C} S_{1}\right)^{n} y_{n}, y_{n}\right)=0$. Since $\phi\left(S_{1}\left(\Pi_{C} S_{1}\right)^{n} y_{n}, S_{1} y_{n}\right) \leq$ $k_{1} \phi\left(\left(\Pi_{C} S_{1}\right)^{n} y_{n}, y_{n}\right)$, then

$$
\lim _{n \rightarrow \infty} \phi\left(S_{1}\left(\Pi_{C} S_{1}\right)^{n} y_{n}, S_{1} y_{n}\right)=0 .
$$

Applying (3.12), (3.14), the definition of $S_{1}$, we obtain that

$$
\lim _{n \rightarrow \infty} \phi\left(y_{n}, S_{1} y_{n}\right)=0
$$


From Lemma 2.3, we obtain that

$$
\lim _{n \rightarrow \infty}\left\|y_{n}-S_{1} y_{n}\right\|=0
$$

In the same way, we can obtain

$$
\lim _{n \rightarrow \infty}\left\|y_{n}-S_{i} y_{n}\right\|=0,2 \leq i \leq N .
$$

Choose a subsequence $\left\{x_{n_{i}}\right\}$ of $\left\{x_{n}\right\}$ such that

$$
\limsup _{n \rightarrow \infty}\left\langle\left(1-\alpha_{n}\right) x_{n}-\bar{x}, J \bar{x}\right\rangle=\lim _{i \rightarrow \infty}\left\langle\left(1-\alpha_{n}\right) x_{n_{i}}-\bar{x}, J \bar{x}\right\rangle .
$$

As $\left\{x_{n_{i}}\right\}$ is bounded, without loss of generality that $x_{n_{i}} \rightarrow z$. From (3.11), we have that $y_{n_{i}} \rightarrow z$. From (3.17) and $I-S_{i}$ is demi-closed, we have $z \in$ $\cap_{i=1}^{N} F\left(S_{i}\right), 1 \leq i \leq N$. Then by Lemma 2.2 , we immediately obtain that

$$
\limsup _{n \rightarrow \infty}\left\langle\left(1-\alpha_{n}\right) x_{n}-\bar{x}, J \bar{x}\right\rangle=\lim _{i \rightarrow \infty}\left\langle\left(1-\alpha_{n}\right) x_{n_{i}}-\bar{x}, J \bar{x}\right\rangle=\langle z-\bar{x}, J \bar{x}\rangle \geq 0 .
$$

Then from (3.5), we get that

$$
\phi\left(\bar{x}, x_{n+1}\right) \leq\left(1-\theta_{n}\right) \phi\left(\bar{x}, x_{n}\right)-2 \theta_{n}\left\langle\left(1-\alpha_{n}\right) x_{n}-\bar{x}, J \bar{x}\right\rangle+\left(k_{n}-1\right) M .
$$

Note that $\lim _{n \rightarrow \infty} \theta_{n}=0, \sum_{n=1}^{\infty} \theta_{n}=\infty$. From Lemma 2.6 that $\lim _{n \rightarrow \infty} \phi\left(\bar{x}, x_{n}\right)=$ 0 . Consequently, from Lemma $2.3, x_{n} \rightarrow \bar{x}, n \rightarrow \infty$.

Case 2. Suppose that there exists a subsequence $\left\{n_{i}\right\}$ of $\{n\}$ such that

$$
\phi\left(\bar{x}, x_{n_{i}}\right) \leq \phi\left(\bar{x}, x_{n_{i}+1}\right)
$$

for all $i \in \mathbf{N}$. Then by Lemma 2.7, there exists a nondecreasing sequence $\left\{m_{k}\right\} \subset$ $\mathbf{N}$ such that $m_{k} \rightarrow \infty, \phi\left(\bar{x}, x_{m_{k}}\right) \leq \phi\left(\bar{x}, x_{m_{k}+1}\right)$, and $\phi\left(\bar{x}, x_{k}\right) \leq \phi\left(\bar{x}, x_{m_{k}+1}\right)$ for all $k \in \mathbf{N}$. Then from (3.5) and $\lim _{n \rightarrow \infty} \theta_{n}=0$, we have

$$
\begin{aligned}
& \sum_{i=1}^{N} \beta_{m_{k}, 0} \beta_{m_{k}, i} g\left(\left\|J x_{m_{k}}-J S_{i}\left(\Pi_{C} S_{i}\right)^{m_{k}-1} y_{m_{k}}\right\|\right) \\
& \leq \phi\left(\bar{x}, x_{m_{k}}\right)-\phi\left(\bar{x}, x_{m_{k}+1}\right)-\theta_{m_{k}} \phi\left(\bar{x}, x_{m_{k}}\right)-2 \theta_{m_{k}}\left\langle\left(1-\alpha_{m_{k}}\right) x_{m_{k}}-\bar{x}, J \bar{x}\right\rangle \\
& +\left(k_{m_{k}}-1\right) M .
\end{aligned}
$$

This implies that $g\left(\left\|J x_{m_{k}}-J S_{i}\left(\Pi_{C} S_{i}\right)^{m_{k}-1} y_{m_{k}}\right\|\right) \rightarrow 0$ as $n \rightarrow \infty$. Thus, following the method of case 1 , we obtain that $\left\|x_{m_{k}}-y_{m_{k}}\right\| \rightarrow 0$ and $\| y_{m_{k}}-$ $S_{i} y_{m_{k}} \| \rightarrow 0$ as $n \rightarrow \infty, \forall 1 \leq i \leq N$. Hence there exists $\dot{z} \in F$ such that

$$
\limsup _{n \rightarrow \infty}\left\langle\left(1-\alpha_{m_{k}}\right) x_{m_{k}}-\bar{x}, J \bar{x}\right\rangle=\langle\dot{z}-\bar{x}, J \bar{x}\rangle \geq 0 .
$$

Then from (3.5), we get that

$\phi\left(\bar{x}, x_{m_{k}+1}\right) \leq\left(1-\theta_{m_{k}}\right) \phi\left(\bar{x}, x_{m_{k}}\right)-2 \theta_{m_{k}}\left\langle\left(1-\alpha_{m_{k}}\right) x_{m_{k}}-\bar{x}, J \bar{x}\right\rangle+\left(k_{m_{k}}-1\right) M$. 
Since $\phi\left(\bar{x}, x_{m_{k}}\right) \leq \phi\left(\bar{x}, x_{m_{k}+1}\right),(3.20)$ implies that

$$
\begin{aligned}
& \theta_{m_{k}} \phi\left(\bar{x}, x_{m_{k}}\right) \\
& \leq \phi\left(\bar{x}, x_{m_{k}}\right)-\phi\left(\bar{x}, x_{m_{k}+1}\right)-2 \theta_{m_{k}}\left\langle\left(1-\alpha_{m_{k}}\right) x_{m_{k}}-\bar{x}, J \bar{x}\right\rangle+\left(k_{m_{k}}-1\right) M \\
& \leq-2 \theta_{m_{k}}\left\langle\left(1-\alpha_{m_{k}}\right) x_{m_{k}}-\bar{x}, J \bar{x}\right\rangle+\left(k_{m_{k}}-1\right) M .
\end{aligned}
$$

In particular, since $\theta_{m_{k}}>0$, we have that

$$
\phi\left(\bar{x}, x_{m_{k}}\right) \leq-2\left\langle\left(1-\alpha_{m_{k}}\right) x_{m_{k}}-\bar{x}, J \bar{x}\right\rangle+\frac{\left(k_{m_{k}}-1\right)}{\theta_{m_{k}}} M .
$$

Thus, from (3.19) and the fact that $\frac{\left(k_{m_{k}}-1\right)}{\theta_{m_{k}}} \rightarrow 0$, we obtain that $\phi\left(\bar{x}, x_{m_{k}}\right) \rightarrow 0$ as $k \rightarrow \infty$ This together with (3.20) gives $\phi\left(\bar{x}, x_{m_{k}+1}\right) \rightarrow 0$ as $k \rightarrow \infty$. But $\phi\left(\bar{x}, x_{k}\right) \leq \phi\left(\bar{x}, x_{m_{k}+1}\right)$ for all $k \in N$, thus we obtain that $x_{k} \rightarrow \bar{x}$ as $k \rightarrow \infty$. Therefore, from the above that two cases, we can conclude that $\left\{x_{n}\right\}$ converges strongly to the minimum norm point of $F$.

Remark 3.2

(1) Theorem 3.1 extends the results of [5] from a finite family of asymptotically nonexpansive self-mappings to a finite family of asymptotically nonextensive nonself-mappings.

(2) Theorem 3.1 extends the results of [5] from real Hilbert spaces to uniformly smooth and uniformly convex Banach spaces.

\section{References}

[1] F. E. Browder, Convergence theorems for sequences of nonlinear operators in Banach spaces, Math Z, 100 (1967), 201-225. http://dx.doi.org/10.1007/bf01109805

[2] B. Halpern, Fixed points of nonexpanding maps, Bull Am Math Soc, 73 (1967), 957-961. http://dx.doi.org/10.1090/s0002-9904-1967-11864-0

[3] X. Yang, Y. C. Liu and Y. Yao, Finding minimum norm fixed point of nonexpansive mappings and applications, Mathematical problems in Engineering, 2011, Article ID 106450, 13 pages, 2011. http://dx.doi.org/10.1155/2011/106450

[4] Y. H. Yao, H. K. Xu, Iterative methods for finding minimum-norm fixed points of nonex-pansive mappings with applications, Optim, 60 (6) (2011), 645-658. http://dx.doi.org/10.1080/02331930903582140

[5] H. Zegeye, N. Shahzad, Approximation of the common minimumnorm fixed point of finite family of asymptotically nonexpansive mappings, Fixed Point Theory of Applications, 2013, 2013: 1. http://dx.doi.org/10.1186/1687-1812-2013-1 
[6] K. Goebel, W. A. Kirk, A fixed point theorem for asymptotically nonexpansive mappings, Proc. Amer. Math. Soc, 35 (1972), 171-174. http://dx.doi.org/10.1090/s0002-9939-1972-0298500-3

[7] C. E. Chidume, M. Khumalo, and H. Zegeye, Generalized projection and approximation of fixed points of nonself maps, Journal of Approximation Theory, 120 (2003), 242-252. http://dx.doi.org/10.1016/s00219045(02)00021-7

[8] Y. Liu, Convergence theorems for common fixed points of nonself asymptotically nonextensive mappings, J Optim Theory Appl, 162 (2014), 79-87. http://dx.doi.org/10.1007/s10957-013-0402-9

[9] Y. I. Alber, Metric and generalized projection operators in Banach spaces: properties and applications, in:Kartsatos A. (Eds.) Theory and Applications of Nonlinear Operators of Monotonic and Accretive Type, pp.15-50, Marcel Dekker, New York, 1996.

[10] S. Matsushita, W. Takahashi, A strong convergence theorem for relatively nonexpansive mappings in a Banach space, Journal of Approximation Theory, 134 (2005), 257-266. http://dx.doi.org/10.1016/j.jat.2005.02.007

[11] H. K. Xu, Inequalities in Banach spaces with applications, Nonlinear Analysis, Theory, Methods and Applications, 16 (1991), 1127-1138. http://dx.doi.org/10.1016/0362-546x(91)90200-k

[12] H. K. Xu, An iterative approach to quadratic optimization, J Optim Theory Appl, 116 (2003), 659-678. http://dx.doi.org/10.1023/a:1023073621589

[13] P. E. Manigé, Strong convergence of projected subgradient methods for nonsmooth and nonstrictly convex minization, Set-Valued Anal, 16 (2008), 899-912. http://dx.doi.org/10.1007/s11228-008-0102-z

Received: December 3, 2014; Published: December 30, 2014 